\title{
Electrogastrography: a document prepared by the gastric section of the American Motility Society Clinical GI Motility Testing Task Force
}

\author{
H. P. PARKMAN, ${ }^{\star}$ W. L. HASLER,$\dagger$ J. L. BARNETT $\dagger \&$ E. Y. EAKER $\ddagger$ \\ ${ }^{\star}$ GI Section, Department of Medicine, Temple University Hospital, Philadelphia, PA, USA \\ $\dagger$ Division of Gastroenterology, Department of Internal Medicine, University of Michigan Medical Center, Ann Arbor, MI, USA \\ †Division of Gastroenterology, Department of Medicine, University of Kansas Medical Center, KS, USA
}

\begin{abstract}
The objective of this document is to present the consensus opinion of the American Motility Society Clinical GI Motility Testing Task Force on the performance and clinical utility of electrogastrography (EGG). EGG is a non-invasive means of recording human gastric myoelectrical activity or slow waves from cutaneous leads placed over the stomach. In healthy volunteers, EGG tracings exhibit sinusoidal waveforms with a predominant frequency of 3 cycles per minute (cpm). Clinical studies have shown good correlation of these cutaneous recordings with those acquired from serosally implanted electrodes. The amplitude of the EGG waveform increases with ingestion of caloric or non-caloric meals. Some patients with nausea, vomiting, or other dyspeptic symptoms exhibit EGG rhythm disturbances or blunting of meal-evoked EGG signal amplitude increases. These abnormalities correlate to some degree with delayed gastric emptying of solids. In selected patients, EGG may be complementary to gastric emptying testing. To date, no therapies have convincingly demonstrated in controlled studies that correcting abnormalities detected by EGG improves upper gastrointestinal symptoms. Proposed clinical indications for performance of EGG in patients with unexplained nausea, vomiting and dyspeptic symptoms must be validated by prospective controlled investigations.
\end{abstract}

Address for correspondence

Henry P. Parkman, MD, GI Section, Department of Medicine, Temple University Hospital, 3401 North Broad Street, Philadelphia, PA 19140, USA.

Tel.: +215 707 7579; fax: +215 707 2684;

e-mail: hparkman@nimbus.temple.edu

Received: 9 April 2002

Accepted for publication: 20 December 2002
Keywords electrogastrography, gastric slow wave, gastric emptying, nausea and vomiting.

\section{INTRODUCTION}

Electrogastrography (EGG) is a technique for recording gastric myoelectrical activity using cutaneous electrodes placed on the anterior abdominal wall overlying the stomach. Studies have shown good correlation between cutaneous EGG recordings and myoelectric signals recorded from gastric serosal leads. ${ }^{1-4}$ EGG has been advocated as a diagnostic test for the clinical evaluation of patients with unexplained nausea, vomiting and other dyspeptic symptoms to gain insight into mechanisms of symptom generation. This document presents the consensus opinion of the American Motility Society (AMS) Clinical GI Motility Task Force on EGG. This document is intended to serve as a reference for clinical EGG testing and will present information on: (i) normal gastric myoelectric physiology, (ii) slow wave abnormalities in patients with nausea and vomiting (iii) performance of EGG, (iv) interpretation of EGG recordings, (v) clinical importance of EGG testing including proposed indications, and (vi) recommendations for future investigation to clarify a role for EGG in the evaluation and management of patients with nausea and vomiting.

\section{NORMAL AND ABNORMAL GASTRIC MYOELECTRIC PHYSIOLOGY}

Gastric electrical pacemaker activity originates on the greater curvature at the junction between the proximal and distal stomach. Slow waves, rhythmic electrical depolarizations occurs at a frequency of 3 cycles per minute $(\mathrm{cpm})$ in humans. It is believed that the interstitial cells of Cajal generate the rhythmic 
depolarizations of the gastric slow wave. Under quiescent conditions, slow waves partially depolarize gastric smooth muscle but do not cause contraction. Additional depolarizations provided by neurohumoral stimulation are the triggers for phasic gastric contractions which follow the spread of the electrical slow waves and are peristaltic. Thus, gastric electrical slow waves control the maximal frequency and the direction of contractions in the distal stomach.

As with the heart, abnormal pacemaker activity and electrical activity in other gastric regions may replace the normal $3 \mathrm{cpm}$ rhythm produced by the physiological pacemaker region. ${ }^{5}$ Bradygastria develops when depolarizations occur at frequencies lower than normal. With bradygastria, the contractile efficiency of the stomach is reduced as a result of decrease in the number of antral contractions. Tachygastria develops when an ectopic pacemaker, often in the antrum, generates an oscillatory pattern at an abnormally high frequency that overdrives the rest of the stomach. Although retrograde depolarizations may propagate with tachygastria, retrograde motor activity rarely develops as the electrical activity is of insufficient amplitude to induce contraction. Thus, during tachygastria, the stomach is usually atonic. In some instances, the ectopic pacemaker activity is unstable in frequency and in location, leading to mixed gastric tachy-bradydysrhythmia. Each of these rhythms may be present for very short periods of time in healthy humans and dogs, but pathological conditions may be associated with prolonged periods of slow wave rhythm disruption. ${ }^{6,7}$

\section{SLOW WAVE ABNORMALITIES IN PATIENTS WITH NAUSEA AND VOMITING}

Many clinical conditions which cause delayed gastric emptying also are associated with abnormalities of EGG frequency and the amplitude response to a meal. Tachygastria and bradygastria are found in many patients with diabetic gastroparesis. ${ }^{8}$ In addition to disturbances of EGG rhythm, some diabetics exhibit a concurrent loss of the signal amplitude increase with meal ingestion. ${ }^{9}$ Furthermore, hyperglycaemia is associated with increased dysrhythmic activity. ${ }^{10}$ Conversely, one investigation noted no increase in slow wave rhythm disturbances in diabetic patients in whom euglycaemia was maintained, although defects in meal-related amplitude responses persisted. ${ }^{11}$ Dysrhythmias also are prevalent in patients with gastro-oesophageal reflux and delayed gastric emptying. ${ }^{12}$ In one report of nausea of first trimester pregnancy, EGG rhythm disturbances were observed in 26 of 32 women. ${ }^{13}$ Dysrhythmias were documented in 13 of 14 patients with chronic intestinal pseudoobstruction secondary both to visceral myopathy and neuropathy. ${ }^{14}$ Conversely, no EGG abnormalities were reported in scleroderma patients with dyspepsia. ${ }^{15}$ Patients with anorexia nervosa and delayed gastric emptying exhibit frequent dysrhythmic episodes, however a recent report of patients with early onset anorexia nervosa showed no increase in dysrhythmias suggesting that EGG rhythm disorders do not initiate the syndrome. ${ }^{16,17}$ Other disorders associated with gastric dysrhythmias include motion sickness and gastroparesis caused by ischaemia, chronic renal failure and occurring as a paraneoplastic syndrome. ${ }^{18,19}$

In addition to these well-characterized clinical conditions, many patients with unexplained nausea and vomiting exhibit slow wave disturbances. Gastric dysrhythmias have been observed in $31-69 \%$ of patients with functional dyspepsia. ${ }^{20,21}$ Among dyspeptic patients, EGG rhythm disturbances occur in $75 \%$ of patients with delayed gastric emptying vs $25 \%$ of dyspeptic individuals with normal emptying. ${ }^{21}$ The association of EGG dysrhythmias with delays in gastric emptying in some patients with idiopathic gastroparesis suggest a possible pathogenic role for these primary slow wave rhythm disruptions. ${ }^{22}$ Finally, studies have identified patients with unexplained nausea and vomiting without abnormalities of gastric emptying who exhibit either EGG dysrhythmias or abnormal amplitude responses to meal ingestion. ${ }^{23,24}$

\section{PERFORMANCE OF EGG}

\section{Equipment}

Several components are necessary for the performance of EGG. The EGG signals are acquired using electrocardiographic electrodes affixed to the skin of the abdominal wall. Pregelled adhesive $\mathrm{Ag} / \mathrm{AgCl}$ electrodes frequently are employed because they facilitate reliable acquisition of the cutaneous signal for the duration of a typical clinical study. Amplifiers and filters are needed to process the EGG signal for subsequent analysis; usually these are in one unit. Amplifiers are required because of the relatively weak gastric signal acquired by the cutaneous electrodes $(200-500 \mu \mathrm{V})$. Low and high band pass filters at 0.016 and $0.3 \mathrm{~Hz}$, respectively, help to eliminate baseline drift, exclude signals from other sources (heart, small intestine and some colonic frequencies), and remove some artefacts occurring as a consequence of respiration and movement. Some centres employ pneumography to 
aid in the identification of respiration and motion artefacts. Per regulatory requirements, all components of the EGG recording system must be electrically isolated from the individual being studied to ensure unidirectional current flow from the skin to the amplifiers. All equipment including the computer is plugged into an isolated medical grade isolation transformer. Some institutions use a signal generator to calibrate the EGG signal against a test sinusoidal wave of known frequency. Vendors who currently offer or have offered EGG equipment in the past include 3CPM Company, Sandhill Scientific, Inc., Medtronic/Synectics, RedTech, and MMS.

Computers employed for EGG analysis must possess specific characteristics. An analogue to-digital (A-D) board is needed to digitize the signal at frequencies ranging from 1 to $4 \mathrm{~Hz}$. Software has been developed for preprocessing excision of motion or respiratory artefacts. Signal analysis is performed with Fast Fourier transformation (FFT) and running spectral analysis (RSA) or adaptive running spectral analysis. Data commonly are presented in the form of a RSA in which results from FFT of consecutive or overlapping recording periods are presented in three-dimensional format to demonstrate frequency vs power peaks as a function of time.

\section{Test protocol}

Patient preparation As with other tests of gastric function, EGG recordings most often are performed after overnight fasting. Some institutions have advocated ingestion of a pretest meal including four ounces of apple juice and one slice of toast $2 \mathrm{~h}$ before testing to enhance detection of the basal waveform, however this practice does not permit recording of a true fasting signal. Medications that might modify gastric myoelectrical activity (prokinetic and anti-emetic agents, narcotic analgesics, anticholinergic drugs, non-steroidal antiinflammatory agents) should be stopped at least $48 \mathrm{~h}$ prior to testing. ${ }^{25}$ Other medications which influence slow wave activity, such as anti-depressants and oral contraceptives, may be difficult to stop for the purposes of EGG recording. Hyperglycaemia in excess of $230 \mathrm{mg} / \mathrm{dL}^{-1}$ has been demonstrated to disrupt normal EGG rhythm in healthy volunteers. ${ }^{26}$ Furthermore, normalization of blood glucose levels prior to testing reduces gastric dysrhythmic activity in patients with type I diabetes. ${ }^{11}$ For these reasons, it is reasonable to recommend performance of EGG under conditions of euglycaemia to better gauge underlying gastric myoelectric properties in the absence of modulating metabolic factors.
EGG recording The EGG should be recorded in a quiet room to minimize extraneous electrical signals which might be detected by the cutaneous electrodes and reduce distractions which could promote patient movement. The patient may be placed in any conformation ranging from a supine position to a $45^{\circ}$ inclination, as long as the patient's position and comfort are maintained for the duration of testing. Changes in position can alter EGG parameters relating to signal amplitude, thus the patient should remain motionless for the duration of the recording. ${ }^{22,27}$ Use of the restroom is encouraged prior to recording to minimize the need for interruption of the test and to eliminate any motion artefacts which might develop as a consequence of urinary or faecal urgency.

Proper EGG electrode placement is important to ensure acquisition of a high quality gastric myoelectric signal. Any hair on the skin overlying the stomach should be shaved to improve conduction. The skin should be gently abraded with gauze or a specific electrode paste (Nuprep; DO Weaver, Aurora, CA, USA). Fresh disposable electrocardiographic electrodes that adhere securely to the skin are employed (Cleartrace; ConMed Corp., Utica, NY, USA) often in concert with an electrode cream (Signa Creme; Parker Laboratories, Orange, NJ, USA) to improve signal transmission.

Several different electrode placements have been described to reliably record gastric myoelectrical activity (Fig. 1). Use of a bipolar electrode system is

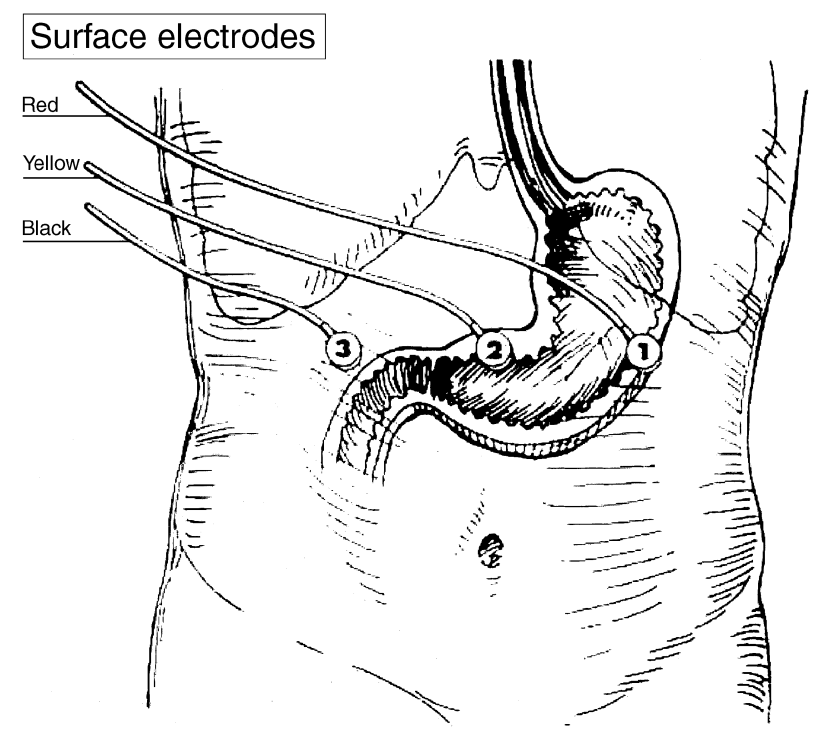

Figure 1 One commonly used electrode placement for electrogastrography recording is shown. Active electrodes are positioned below the left costal margin and, between the xyphoid process and umbilicus. The electrode positioned in the right upper quadrant is a reference or common electrode. 
recommended to reduce artefact and improve the signal-to-noise ratio. To enhance signal acquisition, active recording electrodes are placed along the antral axis of the stomach. A commonly employed configuration involves placement of one active electrode in the midline halfway between the xiphoid and umbilicus. A second active electrode is placed $5 \mathrm{~cm}$ to the left of the first active electrode, $30^{\circ}$ cephalad, at least $2 \mathrm{~cm}$ below the rib cage, in the midclavicular line. A reference electrode is placed at a site distant to the active electrodes such as the right midclavicular line or the left midaxillary line. The EGG leads should not be placed over the ribs, as this can increase respiratory artefacts in the recorded signal.

After appropriate patient preparation, EGG recording is performed. A fasting signal is acquired for 15-60 min. The patient then consumes a test meal and a stimulated EGG signal is recorded for $30-120 \mathrm{~min}$. The optimal lengths of recording have not been validated in controlled investigations, however it has been observed that longer study durations enhance detection of EGG rhythm disturbances. ${ }^{28,29}$ It was the consensus opinion of the AMS Clinical GI Motility Testing Task Force that a 30-min fasting recording and a 60-min postprandial EGG recording are obtained. A variety of test meals have been employed by different institutions including water, commercially prepared balanced liquid nutritional supplements (Ensure; Ross Products Division, Abbott Laboratories, Columbus, OH, USA) and solid meals similar to those used in gastric emptying testing (e.g., egg sandwich with orange juice). Each meal produces qualitatively similar effects on the EGG signal, although effects of water ingestion on signal amplitude are shorter in duration. ${ }^{30}$ No investigation has determined if any particular meal characteristic improves the sensitivity or specificity of detection of the specific EGG parameters. Some centres have combined EGG recording with measures of gastric perception in the form of the water load test. ${ }^{30}$ No study to date has assessed if distending the stomach to the point of maximal fullness with such satiety testing produces different EGG results than those observed with ingestion of fixed meal volumes.

Symptoms may be quantified either at fixed intervals or with reports of specific symptoms during performance of fasting and postprandial EGG recording. Fullness, bloating, abdominal pain and hunger have been assessed, however the symptoms most commonly associated with EGG rhythm disturbances are nausea and vomiting. ${ }^{25,30}$ Symptoms may be recorded in analogue or integral fashion, however any patient recording methodology should minimize arm motion to decrease disruption of EGG recording.

\section{INTERPRETATION OF EGG RECORDINGS}

\section{Findings provided by EGG}

Analysis of a well-performed EGG examination yields specific findings which can be related to the referring clinician. A normal EGG is characterized by a waveform with a frequency of approximately $3 \mathrm{cpm}$ wave activity that increases in amplitude (or power) after meal ingestion (Fig. 2). Rhythm abnormalities observed with EGG include tachygastria, bradygastria, and a non-specific dysrhythmia or lack of a single dominant frequency (DF) (Fig. 3). The other main EGG abnormality which has been characterized is a lack of signal power increase after eating (Fig. 4). Analysis of a clinical EGG recording involves visual inspection of the raw tracing followed by computer-assisted calculation of dysrhythmic activity and postprandial power responses. Artefacts in the EGG signal must be identified and excluded from computer analysis.

\section{Visual analysis of the EGG recording}

Visual analysis of the raw EGG tracing serves to identify periods of artefact and provide a qualitative determination of tachy- or bradygastric activity and the presence or absence of a signal power increase after eating a test meal (Table 1). Artefacts usually are readily recognized by the interpreter as sudden, highamplitude off-scale deflections of the EGG signal (Fig. 5). In some instances, artefacts exhibit similar waveform characteristics as slow wave dysrhythmias and thus may be a source of interpretational inaccuracy. This source of error is minimized in settings in which the subject undergoing EGG is observed by a technician during recording. In general, such artefacts are caused by respirations, volitional limb or torso movements, retching, or urinary or faecal evacuation. Most motion artefacts exhibit apparent frequencies of $<1 \mathrm{cpm}$ whereas respiratory artefact commonly appears as oscillations at $>9 \mathrm{cpm}$.

Once artefactual periods are excluded, the interpreter makes a qualitative determination of the quality of the EGG representation of the gastric slow wave. In general, a visually interpretable signal exhibits a sinusoidal or near-sinusoidal oscillation for a prolonged period of time. This can include recording segments with normal frequencies of approximately $3 \mathrm{cpm}$ and those of abnormally low (bradygastria) or high (tachygastria) frequencies. Visual signal analysis usually also can ascertain whether the signal amplitude increases after ingestion of the test meal. It was the consensus opinion of the Clinical GI Motility Testing Task Force 
(A)

\section{Fasting}

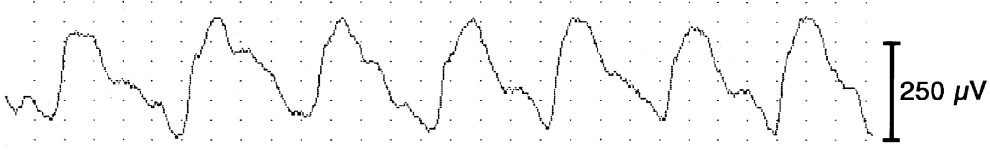

\section{Postprandial}

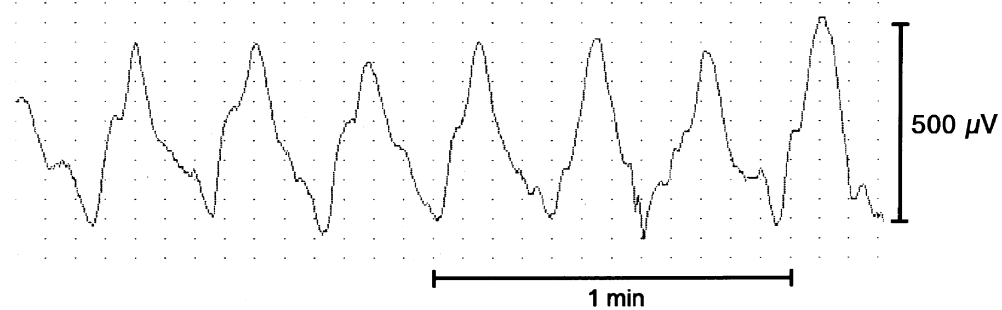

Figure 2 This figure shows the electrogastrography (EGG) tracings and computer analyses from a normal volunteer. (A) The raw tracing demonstrates a sinusoidal oscillation with a frequency of $3 \mathrm{cpm}$ during both the fasting and postprandial periods. Signal amplitude increases with meal ingestion. (B) Running spectral analysis displays the dominant EGG frequencies as a function of time. Throughout the recording, the dominant frequency (DF) is in the frequency band from 2 to $4 \mathrm{cpm}$. (C) The power frequency spectrum displays the DF for the entire fasting and postprandial periods $(3 \mathrm{cpm})$. The increase in power with meal ingestion can be quantified using this analysis. Small peaks at harmonics of the DF are seen at 6 and $9 \mathrm{cpm}$.
(B)

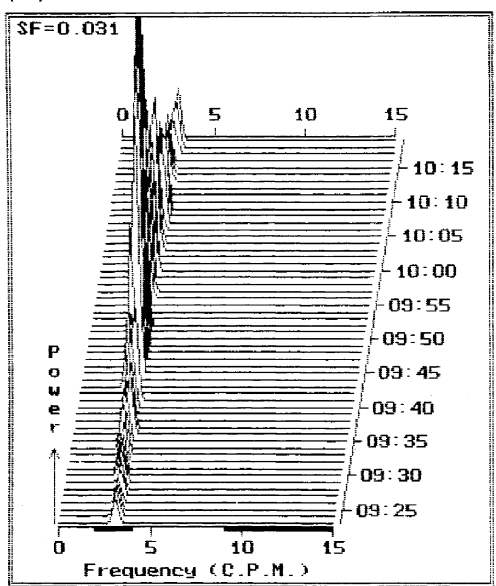

(C)

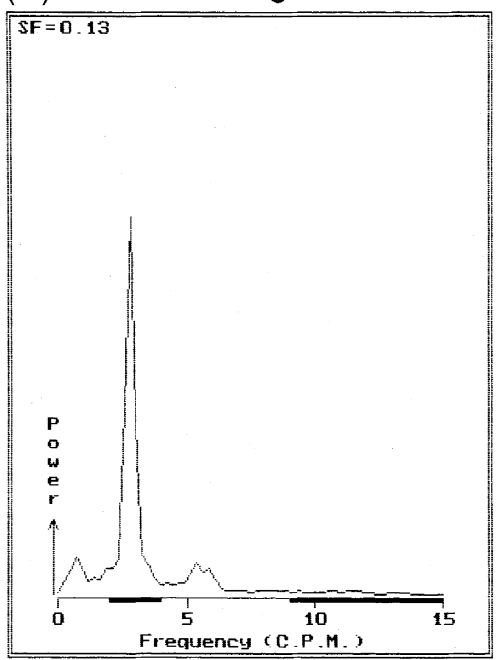

Postprandial

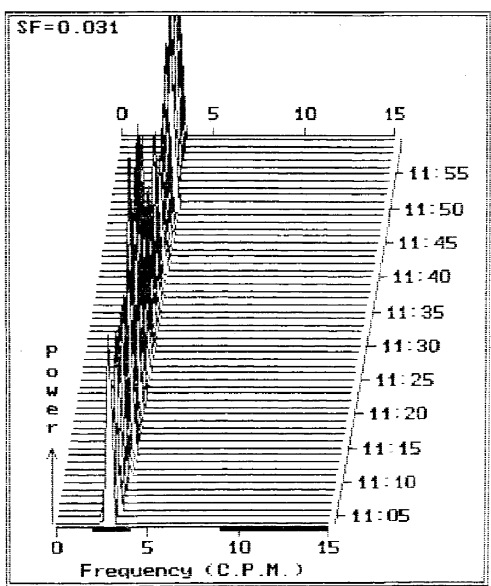

Postprandial

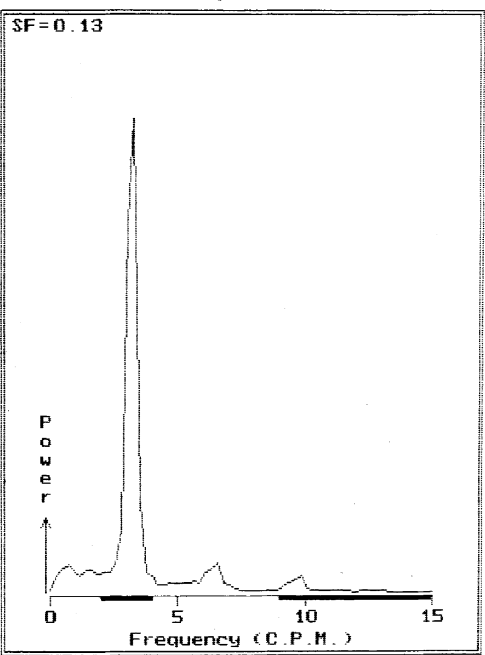




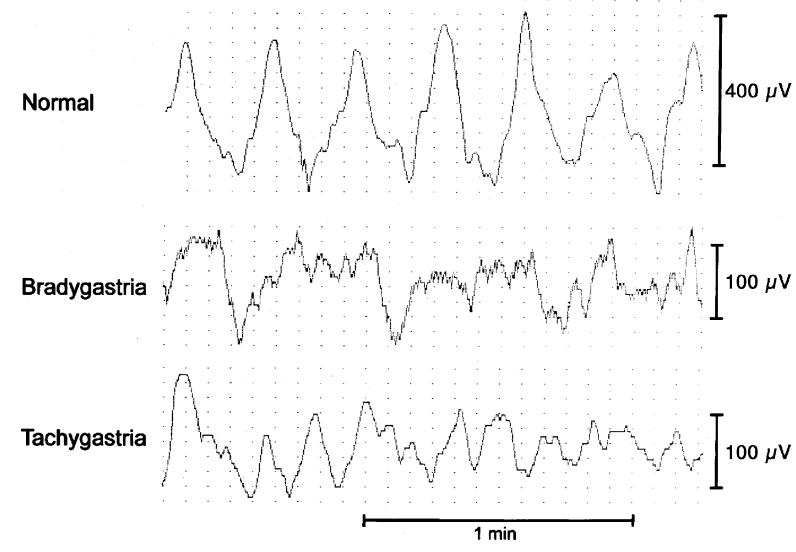

Figure 3 Representative raw electrogastrography tracings showing normal rhythm, bradygastria and tachygastria are shown.

that such recognizable waveforms are visually identifiable for at least $15 \mathrm{~min}$ of the fasting recording and at least $30 \mathrm{~min}$ of the postprandial recording to provide a reliable EGG interpretation. Furthermore, the visual report of a dysrhythmia should be based on determination of an abnormal frequency waveform for at least $5 \mathrm{~min}$. These opinions are based on the extensive clinical and investigative experience of the Task Force members and are not subjected to controlled investigation. In most instances, visual interpretation of the raw EGG signal can provide a preliminary impression of normalcy, tachygastria, bradygastria or a blunted power response to meal ingestion. Computer analysis of the raw signal serves to quantify DFS, lengths of time with dysrhythmia, and the magnitude of the postprandial power increase. The interpreter should exercise care in placing too much weight on computerderived analyses of raw signals in which there are no visually recognizable waveforms.

\section{Computer analysis of the EGG recording}

Prior to subjecting the visually recognizable waveforms to computer analysis, periods which exhibit clear artefact should be excised. Motion artefacts, if included in the quantitative computer software, may lead to erroneous determinations of increased high amplitude, low frequency bradygastric activity (Fig. 5). ${ }^{31,32}$ In general, EGG signals containing artefact are identified by visual inspection and excluded prior to subjecting the signal to computer analysis. Thus, the artefactual segment is not subjected to computer analysis. Some centres excise the artefactual segment and splice back the ends of the non-artefactual signals. Other centres have removed the portion of the RSA after performing the FFT on the artefactual recording segment. The focus of signal analysts was to develop a software which can recognize and automatically remove signal artefacts. Prototypic analytical programs were reported, however they are imperfect and have not achieved widespread acceptance by investigators or clinicians who perform EGG.

After artefacts in the EGG signal were removed, the remainder of the recording is subject to computer analysis. Most institutions employ software that performs FFT on the raw signal with generation of RSA plots, although adaptive RSA techniques were also used and provide equally valid results. One drawback of the FFT with RSA is that EGG dysrhythmias need to be of certain duration to be detected by the calculation. Most EGG analysis programs use recording epochs of approximately 4 min duration for the RSA analysis. Most detectable rhythm disturbances must be 2 min or more in duration. Dysrhythmias of shorter duration may be missed by the FFT with RSA technique. Newer techniques, such as adaptive RSA, may detect shorter duration dysrhythmias.

Several parameters are calculated from computer analysis of the raw EGG signal. The three most commonly reported computations include: (i) the percentage of recording time with the (DF) in the normal $3 \mathrm{cpm}$, tachygastric and bradygastric frequency ranges, (ii) the percentage distributions of EGG power in the three frequency bands, and (iii) the ratio of postprandial to fasting power of the DF. Other parameters of lesser importance but often calculated with computer software programs include (i) the overall dominant EGG frequency in the fasting and postprandial periods, (ii) instability factors of the dominant frequency (DFIC) for the fasting and postprandial periods, and (iii) instability factors for the dominant power (DPIC) in the fasting period and postprandial periods.

Results of these calculations are dependent on what values are set for the upper and lower limits for the normal, tachygastric and bradygastric frequency ranges. From recordings acquired from serosal or mucosal electrodes, the physiological human slow wave frequency ranges from 2.5 to $3.6 \mathrm{cpm}( \pm \mathrm{SD}) \cdot{ }^{33,34}$ Different institutions have performed computer analysis of raw cutaneous EGG signals using different frequency ranges. Some centers define the normal cutaneous EGG frequency to closely conform to the findings of more direct techniques $(2.5-3.75 \mathrm{cpm})$, while others use broader frequency ranges to define normalcy $(2.0-4.0 \mathrm{cpm}) .{ }^{22}$ Normal frequency ranges employed by commercial EGG equipment manufacturers include 2.5-3.75 cpm (3 cpm), 2.0-4.0 cpm (Medtronic; Shore- 
(A)
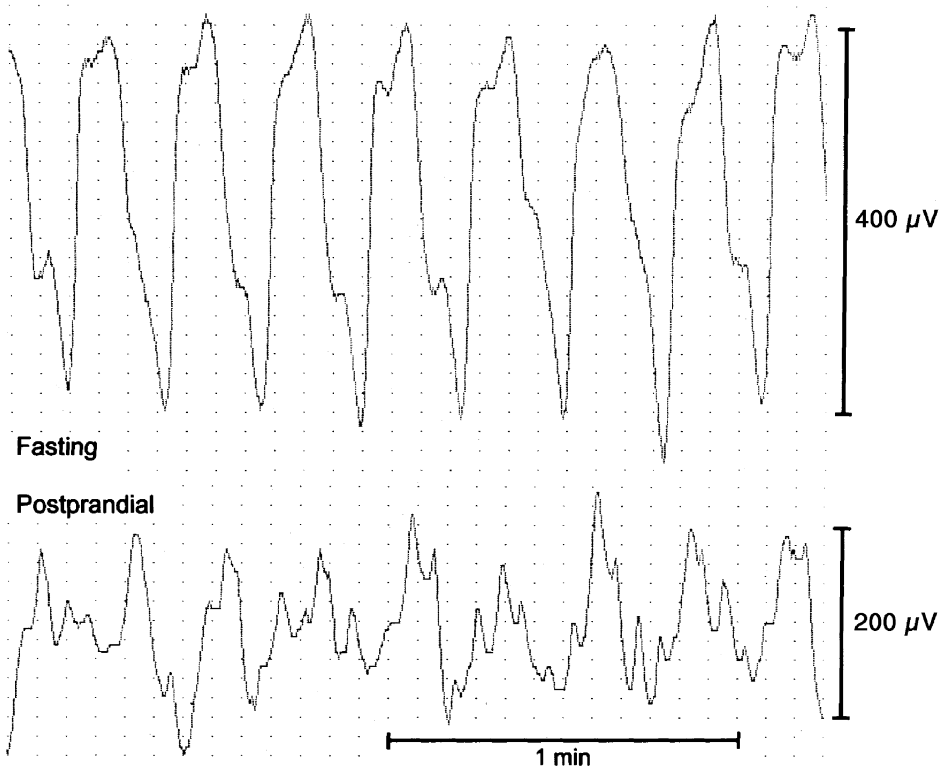

(B)

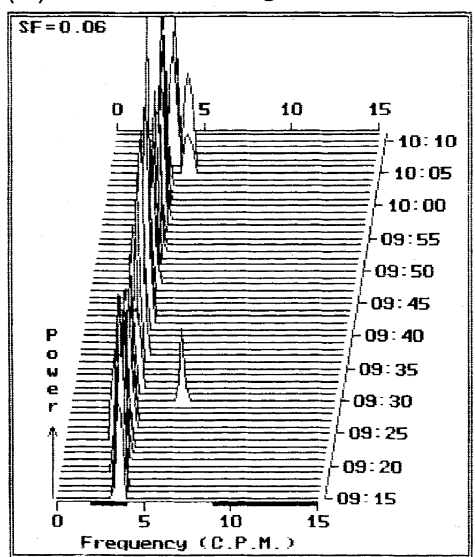

(C)

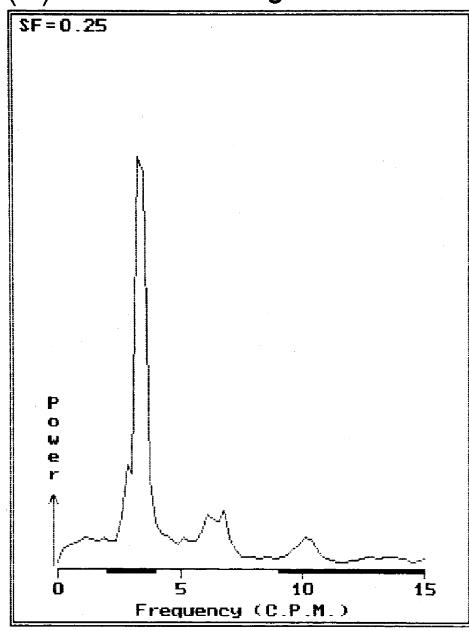

Postprandial

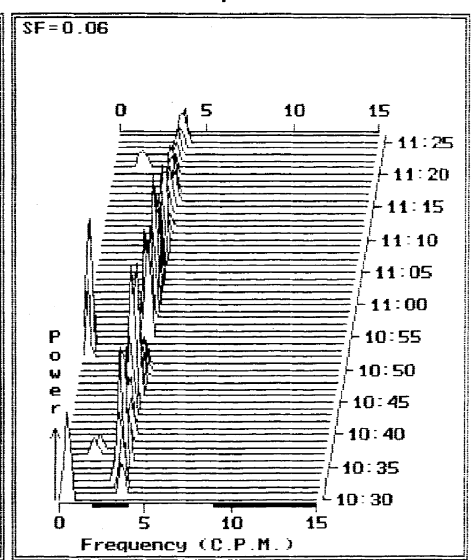

Postprandial

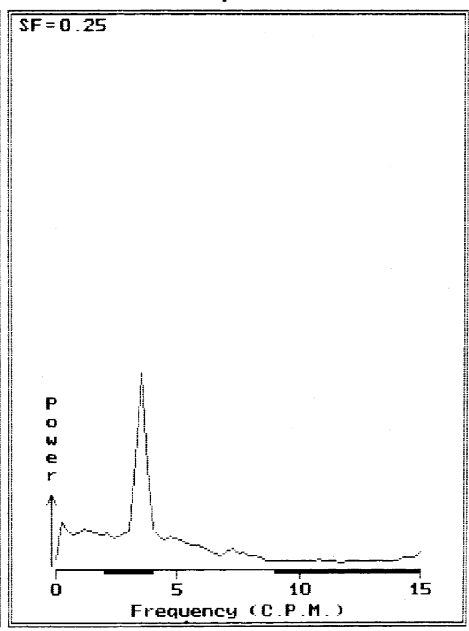

Figure 4 This figure shows the raw tracings and computer analyses in a patient with an impaired electrogastrography (EGG) power response to meal ingestion. (A) The raw signals show normal $3 \mathrm{cpm}$ activity during the fasting and postprandial periods but signal amplitude is decreased after eating. (B) Running spectral analyses and $(\mathrm{C})$ the cumulative power frequency spectrum shows normal EGG frequencies throughout but with blunting of EGG power with meal ingestion. 
Table 1 Analysis of EGG Recordings

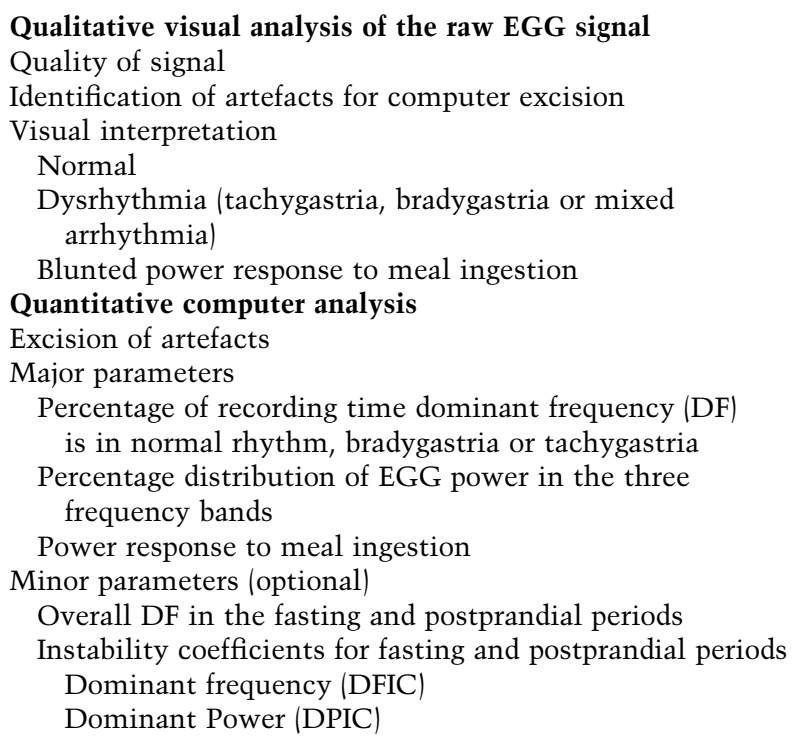

view, MN, USA), and 2.25-3.75 cpm (RedTech, Long Beach, CA, USA). The use of different frequency ranges for computer analysis can produce subtle alterations in the calculations of several computer-derived EGG parameters, especially in determinations of the EGG power distribution in the three frequency bands and the percentage of recording time in the three frequency bands. It is uncertain if broader normal frequency ranges reduce the sensitivity of EGG for detecting dysrhythmic activity with DFs subtly different from normal. It is likely that results from the different analysis protocols will be qualitatively similar. Nevertheless, no rigorous comparisons have been performed comparing the accuracy of different frequency ranges in the detection of abnormal EGG activity. Physicians and investigators performing EGG for clinical diagnosis or research should be cognizant of the definitions of the ranges for normal and abnormal EGG rhythms.

Normal EGG Definitions of what constitutes a normal EGG have been provided by careful analyses of recordings from normal volunteers. In healthy subjects, EGG recordings exhibit normal frequency activity for $\geq 70 \%$ of recording time (Fig. 2). ${ }^{22}$ This determination is based on methodologies which define the normal EGG frequency range as $2.0-4.0 \mathrm{cpm}$. This $70 \%$ value, although initially chosen empirically, generally has been upheld by four studies in 24, 40, 110 and 15 normal subjects. ${ }^{22,35-37}$ In these studies, the lower limit (mean $\pm 2 \mathrm{SD}$ ) of the percentage time that

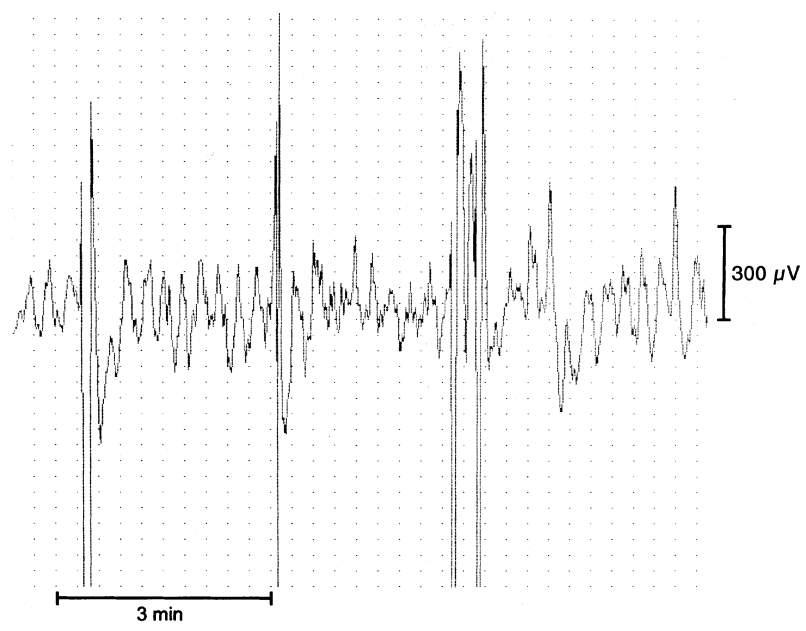

Figure 5 Examples of motion artefacts are shown on this EGG tracing from a healthy volunteer. Large chaotic deflections are observed superimposed on the normal 3 cpm EGG activity. If included in the computer analysis, these artefactual deflections would produce high power signals in the bradygastric frequency range.

normal subjects have a fasting EGG rhythm in the $2.0-4.0 \mathrm{cpm}$ range was $100,58,65$ and $79 \%$, respectively, with a mean of $69 \%$ of the recording time for the 189 subjects in these four studies. For the postprandial period, the lower limit for the percentage time in the $2.0-4.0 \mathrm{cpm}$ range was $100,65,69$ and $78 \%$ with a mean of $73 \%$. Thus, the overall results of these four studies in 189 normal subjects suggest that $70 \%$ is an appropriate lower limit of normal for the percentage of the recording time for the EGG rhythm to be in the $2.0-4.0 \mathrm{cpm}$ range. In such recordings in asymptomatic individuals, the presence of DFs outside the normal range may reflect a low signal amplitude which is obscured by signal noise rather than a true dysrhythmia. ${ }^{25}$ In many instances, this distinction can be made by visual inspection of the raw tracings during such periods. The percentage of time that the postprandial $\mathrm{DF}$ is in the normal frequency also is dependent on specific qualities of the ingested test meal. Solid meals slightly increase EGG frequency, whereas liquid meals temporarily reduce the frequency. ${ }^{22}$ Test meals consisting of an egg sandwich and water $(32 \%$ protein, $46 \%$ carbohydrate, $22 \%$ fat) increase the DF from $2.91 \pm 0.28$ (SD) during fasting to $3.11 \pm 0.28$ in the first postprandial hour. ${ }^{36}$

The percentage distribution of EGG power in the normal, tachygastric and bradygastric frequency bands also can be calculated for both the fasting and postprandial periods and is dependent on the definitions of the different frequency ranges. To calculate the percentage power distribution, the summed signal 
power in each frequency band is divided by the total signal power for the time period being analysed and multiplied by 100 . Normal values from healthy volunteers for power distributions for each frequency range have been published for both caloric and non-caloric liquid meals. ${ }^{30,38}$

Absolute values of EGG power during fasting and the postprandial period are affected by a number of variables including body habitus, electrode placement, and body position, all of which modify the distance from the stomach to the recording leads. ${ }^{22,27}$ However, these variables do not influence the relative increase in EGG power which is observed with ingestion of a caloric meal in healthy subjects (Fig. 2). ${ }^{8,39}$ Depending on the meal consumed, $90-95 \%$ of healthy volunteers exhibit increased postprandial signal power of dominant frequency. ${ }^{22,36}$ In one study, all 24 normal subjects had a postprandial increase in the power of the dominant frequency. ${ }^{22}$ In another study, 90\% of 110 normal subjects had a postprandial increase in the power of the dominant frequency. ${ }^{36}$

Abnormal EGG A variety of abnormalities were described on EGG recordings from patients with nausea and vomiting. Visual inspection of the raw EGG signal can detect the presence of tachygastria (visible oscillation with frequency above the normal range), bradygastria (visible oscillation with frequency below the normal range), a non-specific rhythm (absence of a single predominant rhythm) and a lack of signal increase after meal ingestion (Fig. 3). Computer analysis of the raw EGG signal can quantify the degree of rhythm disturbance and the magnitude of the power increase (Fig. 4). However, the interpreter must be careful in diagnosing tachygastria or bradygastria when no clear-cut high or low frequency waveform is visible on the raw EGG tracing.

Definitions of EGG abnormalities were largely determined by consensus opinions of investigators with extensive experience in the technique. However, comparisons of EGG findings in healthy volunteers and symptomatic patients provide support for these definitions. What constitutes dysrhythmia in a given recording is determined by the definition of normal frequency. Upon computer analysis, an EGG is considered to be abnormal if the DF is in the tachy- and/or bradygastric frequency ranges for more than $30 \%$ of the time. ${ }^{22}$ This number takes into account the observation that healthy volunteers exhibit periods of time representing up to $30 \%$ of recording time in which recognizable EGG rhythms are not distinguishable from background electrical noise either on visual inspection or computer analysis.
Some institutions have advocated the use of percentage distribution of EGG power in the three frequency bands to facilitate detection of dysrhythmic activity. For this parameter, an EGG is considered abnormal if the percentage distribution of total EGG power in the tachygastric range is greater than $20 \% .{ }^{38,40}$ Power distributions in the bradygastric frequency range are highly variable and may be affected by minor variations in the signal baseline or subtle motion artefacts. Thus, the calculation of the percentage of the total EGG power in the bradygastric frequency range may not be reliable for determination of bradygastria.

The final major EGG abnormality is the lack of a signal power increase after meal ingestion. The power ratio, defined as the ratio of the postprandial to fasting power of the DF, normally is greater than one and postulated to reflect a postprandial increase in the electromechanical activity of the stomach with an additional component of gastric distention occurring after meal ingestion. ${ }^{41} \mathrm{~A}$ power ratio of $<1$ is believed to correlate with a decreased distal gastric motor response to a meal.

\section{Reporting of results}

Performance of EGG for clinical purposes should include an informative procedure report consisting of several key components. Patient-related data should be comprehensive including patient name, identifying numbers and procedure date. A brief history should be provided for identifying the patient's major symptoms and a listing of medications which might influence the results of EGG testing. The EGG recording protocol and analysis methodology should be described. The duration of fasting and postprandial recordings, and also the qualitative and quantitative properties of the test meal should be stated. The recording equipment and software analysis protocol should be characterized, including filtering frequencies used and frequency ranges for definition of bradygastria, normal and tachygastria.

Reports of the results of the EGG analysis should include estimates of the quality of recording and the degree of motion artefact. Characterization of the visual analysis of the EGG tracing should describe the approximate recording times in normal rhythm, tachygastria, bradygastria and non-specific rhythm. Visual inspection can also confirm the presence of a postprandial signal amplitude increase. Results of computer analysis conveys the percentage recording time in each of the frequency ranges, the magnitude of the postprandial power increase, and in some institutions the 
distributions of EGG power in each of the three frequency bands. For each of these parameters, normal values for a healthy volunteer population using similar EGG recording, test meals, frequency bands and software analysis should be provided. Additionally, optional parameters that may be included are the overall DF, and frequency and power instability factors for the fasting and postprandial periods.

The EGG report should conclude with the interpreter's overall assessment of the findings of the study. The six possible clinical interpretations are: (i) normal, (ii) tachygastria, (iii) bradygastria, (iv) non-specific rhythm pattern, (v) lack of postprandial power increase and (vi) uninterpretable waveform secondary to technical problems or excessive motion artefact.

\section{CLINICAL ROLE OF EGG}

Electrogastrography is one of the many tests of gastrointestinal function which were proposed to evaluate patients with unexplained nausea, vomiting and other dyspeptic symptoms. As described above, prominent EGG abnormalities are present in such patient groups whereas healthy volunteers rarely exhibit EGG rhythm or power disturbances. Similarly, significant fractions of patients with nausea and vomiting show delays in gastric emptying on scintigraphic testing or low amplitude or uncoordinated contractions on manometric testing of the antrum and duodenum. ${ }^{42}$ Investigations have attempted to ascertain whether gastric emptying scintigraphy and EGG identify similar patient populations. When the tests are performed concurrently, postprandial dysrhythmias and blunting of postprandial power increases were shown in one study to correlate with delays in solid phase gastric emptying. ${ }^{39}$ When data from several studies are considered, the positive predictive value of an abnormal EGG to predict gastroparesis ranges from 50 to $81 \%$ (average of $65 \%$ ), whereas the accuracy of a normal EGG to predict normal gastric emptying in a symptomatic population ranges from 65 to $100 \%$ (average of $76 \%$ ). ${ }^{37,39,43,44}$ Thus although abnormalities of gastric emptying and EGG are present in overlapping patient subsets, the two tests may define slightly different populations. If each test were shown to be useful in directing patient management, then gastric scintigraphy and EGG could be considered complementary examinations. ${ }^{39,43,45}$

The value of any diagnostic test is determined by its roles in directing therapy or providing prognostic information. To date, very few investigations have been performed to demonstrate a critical role for EGG in patient management. In children with neurologic impairment undergoing fundoplication for gastro-oesophageal reflux, EGG abnormalities predict postoperative feeding difficulties. ${ }^{46}$ A second study employed EGG to document evidence of diffuse gut dysfunction in patients with severe slow transit constipation. ${ }^{47}$ In one study, a persistent high amplitude EGG signal was found in patients with delayed gastric emptying from obstruction whereas patients with idiopathic gastroparesis had gastric dysrhythmias. ${ }^{48}$ Further prospective investigations are needed to validate the clinical utility of EGG.

In several studies, albeit with small number of subjects, symptom responses to drug therapy in dyspeptic patients appear to correlate with resolution of EGG abnormalities. In an investigation of diabetic patients with gastroparesis, reductions in nausea after 6 months of treatment were associated with correction of tachy- and bradygastrias. ${ }^{8}$ Conversely, symptomatically improved individuals exhibited no acceleration of delayed gastric emptying questioning the pathophysiological importance of gastric stasis in producing nausea. Similarly, uncontrolled case studies have observed improvements in EGG dysrhythmias in patients who experienced symptom reductions on cisapride. ${ }^{49,50}$ Other reports suggest that dyspeptic patients with EGG abnormalities may exhibit better symptomatic responses to treatment with prokinetic agents than those with normal EGGs. ${ }^{51,52}$ These findings contrast sharply with many gastric scintigraphy studies, which frequently observe poor correlation of symptom benefit with reversal of gastroparesis. ${ }^{53}$ Controlled clinical studies are needed to determine if symptomatic patients with a particular EGG finding (bradygastria, tachygastria, blunted postprandial power increase or normal recording) are better treated with one therapy over another.

\section{Proposed Indications for EGG}

Given the current paucity of outcomes based investigations into the clinical utility of gastric motor and myoelectric testing, it is difficult to confidently define clinical indications for the performance of any test evaluating gastric motor and/or myoelectrical activity including EGG. ${ }^{31,54}$ The following proposed indications for EGG were reached by discussion and consensus opinion of the members of the AMS Clinical GI Motility Testing Task Force. They will require validation and update as meaningful controlled clinical studies are reported. The EGG is proposed as a diagnostic study to non-invasively record gastric myoelectrical activity in patients with unexplained persistent or episodic symptoms which may be related 
to a gastric motility and/or myoelectrical disorder. EGG can be obtained: (i) to define gastric myoelectric disturbances in patients with nausea and vomiting unexplained by other diagnostic testing or associated with functional dyspepsia and (ii) to characterize gastric myoelectric disturbances associated with documented gastroparesis.

There have been no prospective investigations to validate diagnostic algorithms for the evaluation and management of patients with nausea and vomiting. A reasonable initial approach includes performance of endoscopy or contrast radiography studies and selected blood tests. Once organic disease were excluded, the patient may be offered an empiric trial of an antiemetic or prokinetic medication or alternatively subjected to testing of gastric motor and myoelectric function. Scintigraphic measurement of gastric emptying traditionally were considered to be the initial procedure of choice for this purpose. EGG most commonly is performed after gastric emptying scanning and after trials of medications to reduce nausea and vomiting. Gastric dysrhythmias are documented in some patients with nausea and vomiting, and may represent a pathophysiological mechanism for symptoms. EGG may be particularly applicable for patient subsets in whom a radionuclide-based test would be relatively contraindicated including pregnant women or children.

\section{Coding and billing information for EGG}

The Federal Drug Administration (FDA) approved EGG as a test for patient evaluation in 2000. The FDA statement on EGG concluded that EGG is a noninvasive test for detecting gastric slow waves and is able to differentiate adult patients with normal myoelectric activity from those with bradygastrias and tachygastrias. EGG can be considered as part of a comprehensive evaluation of adult patients with symptoms consistent with gastrointestinal motility disorders.

Current Procedural Terminology (CPT) codes for EGG were published in 2001 for EGG recording in the United States. Code 91132 refers to diagnostic, transcutaneous EGG while 91133 refers to diagnostic, transcutaneous EGG with provocative testing. Published Relative Value Units (RVUs) currently are 0.52 for EGG, diagnostic, transcutaneous, and 0.66 for EGG, diagnostic, transcutaneous, with provocation. International Classification of Diseases (ICD-9) Ninth revision, diagnostic codes for which EGG might be considered an appropriate investigation include 787.01 for nausea with vomiting, 787.02 for nausea alone, 787.03 for vomiting alone, 536.3 for gastroparesis, 536.8 for dyspepsia and 536.9 for unspecified functional disorders of the stomach.

\section{RECOMMENDATIONS FOR FUTURE INVESTIGATION}

It is apparent that many issues need to be addressed before the technique of EGG is considered an important diagnostic test for the evaluation of patients with unexplained nausea, vomiting and other dyspeptic symptoms. While EGG testing may detect gastric dysrhythmias or normal gastric rhythm, data must be collected which demonstrate that EGG has a positive impact on the management of affected patients. Other areas worthy of study include further characterization of the pathophysiology of EGG dysrhythmias, refinements in signal acquisition to reduce concerns about diagnostic inaccuracies caused by artefact and other factors, and development of novel technologies.

Directed clinical investigations are needed to define important clinical roles for EGG. Studies to correlate clinical syndromes with particular EGG patterns will suggest possible aetiological factors in disease induction. Furthermore, it should be ascertained if abnormalities of multiple EGG parameters (e.g. dysrhythmia and blunted power response) are associated with more severe clinical syndromes than recordings with single abnormalities. Correlation of symptom resolution on specific therapy with normalization of EGG disturbances will be supportive of a pathogenic role for slow wave disruption in symptom production. These studies may not confirm a true causal role as it is difficult to determine if the gastric dysrhythmias are causing the symptoms or if the symptoms are inducing the gastric dysrhythmias. The demonstration that symptom resolution results after directed ablation of a dysrhythmic focus may provide the necessary proof that EGG rhythm disturbances are causative of specific clinical syndromes. No current or currently proposed therapy including gastric neurostimulation techniques show the promise to meet this rigorous standard. Nevertheless, investigations are needed which will clarify a position for EGG in the diagnostic evaluation of unexplained nausea and vomiting and/or functional dyspepsia. Prospective studies which demonstrate that therapies which improve symptoms in patients with EGG dysrhythmias have no effect on individuals with normal EGG findings and alternate therapies are efficacious in those with normal but not abnormal EGGs will help define the role of EGG. Interestingly, although gastric emptying scanning has been considered a 'gold standard' for the initial evaluation of 
patients with presumed gastric dysmotility, no outcome studies have been published for scintigraphic methods which meet this standard. Yet, many clinicians appear to find gastric emptying results helpful in diagnosis and management of patients with nausea and vomiting. Similar to gastric emptying, several clinicians have suggested EGG test results helpful in diagnosis and management of these patients. Diagnostic algorithms that include any test of gastric myoelectric and motor function, not just EGG, require more rigorous validation before they can be fully accepted for the evaluation of unexplained nausea and vomiting.

Published research findings have provided a better understanding of the pathophysiology of gastric dysrhythmias. However, a number of areas are worthy of further investigation. It is apparent that conditions such as gastroparesis exhibit slow wave rhythm disturbances that originate in the stomach. Other disorders such as motion sickness exhibit gastric dysrhythmias that occur as a consequence of extragastric central nervous stimulation. In some conditions, the cause of the EGG abnormality is uncertain. In functional dyspepsia, the question arises as to whether dysrhythmic activity develop as a consequence of neuromuscular dysfunction of the stomach or as a peripheral manifestation of a central nervous system process perhaps in association with psychological disturbance. Thus, investigations which correlate the ability of specific interventions to ablate gastric dysrhythmic activity with reductions in symptoms may provide insight into the pathophysiological relevance of the gastric myoelectric rhythm abnormality. This line of study will address the central question of whether dysrhythmias are causative of a particular clinical syndrome or if they are epiphenomena. From a mechanistic standpoint, several neurohumoral and tissue mediators including serotonin, acetylcholine, dopamine, vasopressin, sex steroids and prostaglandins have been demonstrated to participate in EGG rhythm disruption in various experimental models. ${ }^{26,55-59}$ Future studies may ascertain if a common mediator or neural pathway induce specific gastric dysrhythmic activity.

Future modifications in the performance of EGG may be developed to enhance the accuracy and utility of the technique in detecting abnormal slow wave activity. Some investigators have observed fewer rhythm disruptions on serosal recordings than with cutaneous EGG, suggesting that some of the cutaneously acquired dysrhythmias may be artefactual in nature. ${ }^{31,33}$ Improved artefact detection may include refinements in EGG hardware and software. Computer-assisted excision of artefacts has been tried with artificial neural networks, however this method needs to be perfected before it can be widely applied. ${ }^{22}$ The normal values for the frequency ranges and the acceptable limits of percentage of recording time in dysrhythmic frequency bands should continue to be clarified with the ongoing study. Furthermore, the variability of EGG recordings needs to be determined in normals and in patients with dysrhythmias. It remains undefined as to what are the best stimuli to test for EGG dysrhythmias. Choice of stimuli or provocative test may depend on clinical objectives for the EGG test. It is conceivable that selected meal characteristics may prove superior in the elicitation of slow wave abnormalities. One group has employed edrophonium as a stimulus for inducing gastric dysrhythmias in patients with dyspepsia. ${ }^{59}$ Such diagnostic challenge testing with a variety of drugs is worthy of further investigation. It is uncertain if some apparent dysrhythmias on EGG result from true ectopic pacemaker activity or if there is uncoupling of otherwise normal slow wave activity with disrupted or aberrant propagation. ${ }^{60}$ Perfection of multichannel EGG systems may provide insight into this issue. ${ }^{61}$ Furthermore, such methods may provide additional information not currently provided by EGG. For example, in one investigation, patients with functional dyspepsia were observed to have retarded aboral slow wave propagation. ${ }^{62}$ Finally, advances in electrode design and artefact exclusion may make it possible to acquire prolonged EGG recordings in ambulatory fashion. Early investigations into ambulatory EGG systems reported problems with significant signal interference from movements throughout the day. ${ }^{63,64}$ If perfected, such a portable system would offer significant advantages over current studies performed in hospital environments, by obtaining prolonged fasting and postprandial recordings in non-stressful environments during waking hours and during sleep and by correlating symptom reports with concurrent EGG abnormalities.

\section{ACKNOWLEDGMENTS}

We wish to acknowledge the important input of two prominent investigators in the field of EGG, Drs Kenneth L. Koch and S. Narasimha Reddy, who offered constructive advice and critical analysis of this manuscript during its preparation.

\section{REFERENCES}

1 Hamilton JW, Bellahsene BE, Reicherlderfer M, Webster $\mathrm{JH}$, Bass P. Human electrogastrograms - comparison of 
surface and mucosal recordings. Dig Dis Sci 1986; 31: 33-39.

2 Familoni BO, Bowes KL, Kingma YJ, Cote KR. Can transcutaneous recordings detect gastric electrical abnormalities? Gut 1991; 32: 141-6.

3 Chen J, Schirmer BD, McCallum RW. Serosal and cutaneous recordings of gastric myoelectrical activity in patients with gastroparesis. Am J Physiol 1994; 266: G90G98.

4 Lin Z, Chen JDZ, Schirmer BD, McCallum RW. Postprandial response of gastric slow waves: correlation of serosal recordings with the electrogastrogram. Dig Dis Sci 2000; 45: 645-51.

5 Abell TL, Malagelada J-R. Electrogastrography: current assessment and future perspectives. Dig Dis Sci 1988; 33: 982-92.

6 Code CF, Marlett JA. Canine tachygastria. Mayo Clin Proc 1974; 49: 325-32.

7 Stoddard CJ, Smallwood RH, Duthie HL. Electrical arrhythmias in the human stomach. Gut 1981; 22: 705-12.

8 Koch KL, Stern RM, Stewart WR, Vasey MW. Gastric emptying and gastric myoelectrical activity in patients with diabetic gastroparesis: effect of long-term domperidone treatment. Am J Gastroenterol 1989; 84: 1069-75.

9 Mantides A, Stefanides G, Kioulanis J, Tzovaras G, Epanomeritakis E, Xynos E. Cutaneous electrogastrography for the assessment of gastric myoelectrical activity in type I diabetes mellitus. Am I Gastroenterol 1997; 92: 1190-3.

10 Jebbink RJ, Samsom M, Bruijs PP et al. Hyperglycemia induces abnormalities of gastric myoelectrical activity in patients with type I diabetes mellitus. Gastroenterology 1994; 107: 1390-7.

11 Jebbink HJ, Bruijs PP, Bravenboer B, Akkermans LM, vanBerge-Henegouwen GP, Smout AJ. Gastric myoelectrical activity in patients with type I diabetes mellitus and autonomic neuropathy. Dig Dis Sci 1994; 39: 2376-3.

12 Cucchiara S, Salvia G, Borrelli O et al. Gastric electrical dysrhythmias and delayed gastric emptying in gastroesophageal reflux disease. Am J Gastroenterol 1997; 92: 1103-8.

13 Koch KL, Stern RM, Vasey M, Botti JJ, Creasy GW, Dwyer A. Gastric dysrhythmias and nausea of pregnancy. Dig Dis Sci 1990; 35: 961-8.

14 Debinski HS, Ahmed S, Milla PI, Kamm MA. Electrogastrography in chronic intestinal pseudoobstruction. Dig Dis Sci 1996; 41: 1292-7.

15 Pfaffenbach B, Adamek RJ, Hagemann D et al. Effect of progressive systemic sclerosis on antral myoelectrical activity and gastric emptying. $Z$ Gastroenterol 1996; 34: 517-21.

16 Abell TL, Malagelada J-R, Lucas AR et al. Gastric electromechanical and neurohormonal function in anorexia nervosa. Gastroenterology 1987; 93: 958-65.

17 Ravelli AM, Helps BA, Devane SP, Lask BD, Milla PJ. Normal gastric antral myoelectrical activity in early onset anorexia nervosa. Arch Dis Child 1993; 69: 342-6.

18 Liberski SM, Koch KL, Atnip R, Stern RM. Ischemic gastroparesis: resolution after revascularization. Gastroenterology 1990; 99: 252-7.

19 Lin X, Mellow MH, Southmayd L, Pan J, Chen JD. Impaired gastric myoelectrical activity in patients with chronic renal failure. Dig Dis Sci 1997; 42: 898-906.
20 Koch KL, Medina M, Bingaman S, Stern RM. Gastric dysrhythmias and visceral sensations in patients with functional dyspepsia (abstract). Gastroenterology 1992; 102: A469.

21 Parkman HP, Fisher RS. Disorders of gastric emptying. In: Yamada T, ed. Yamada Textbook of Gastroenterology 4th edn. Philadelphia, PA: Lippincott, Williams \& Wilkins, 2003 (in press).

22 Chen J, McCallum RW. Gastric slow wave abnormalities in patients with gastroparesis. Am J Gastroenterol 1992; 87: 477-82.

23 You CH, Lee KY, Chey WY, Menguy R. Electrogastrographic study of patients with unexplained nausea, bloating and vomiting. Gastroenterology 1980; 79: 311-14.

24 Geldof H, Van der Schee EJ, Van Blankenstein M, Grashuis JL. Electrogastrographic study of gastric myoelectrical activity in patients with unexplained nausea and vomiting. Gut 1986; 27: 799-808.

25 Camilleri M, Hasler W, Parkman HP, Quigley EMM, Soffer E. Measurement of gastroduodenal motility in the GI laboratory. Gastroenterology 1998; 115: 747-62.

26 Hasler WL, Soudah HC, Dulai G, Owyang C. Mediation of hyperglycemia-evoked gastric slow wave dysrhythmias by endogenous prostaglandins. Gastroenterology 1995; 108: 727-36.

27 Sanaka MR, Xing JH, Soffer EE. The effect of body posture on electrogastrogram. Am I Gastroenterol 2001; 96: S73. (abstract)

28 Guo J-P, Bonapace ES, Parkman HP, Fisher RS. The value of the second postprandial hour during electrogastrography (EGG) in symptomatic patients with and without gastroparesis. Dig Dis Sci 1999; 44: 2150. (abstract)

29 Lin ZY, Sarosiek I, Denton S, Loftiss M, McCallum RW. Gastric dysrhythmia during the second postprandial hour correlates with gastrointestinal symptoms in patients with gastroparesis. Dig Dis Sci 2000; 45: 1250. (abstract)

30 Koch KL, Hong S-P, Xu L. Reproducibility of gastric myoelectric activity and the water load test in patients with dysmotility-like dyspepsia symptoms and in control subjects. J Clin Gastroenterol 2000; 31: 125-9.

31 Verhagen MAMT, van Schelven LJ, Samsom M, Smout AJPM. Pitfalls in the analysis of electrogastrographic recordings. Gastroenterology 1999; 117: 453-60.

32 Levy J, Riley R, Thomas S, Lazar J. An analysis of motion artifacts recorded during surface electrogastrography: are the patterns reproducible? Dig Dis Sci 2000; 45: 1248. (abstract).

33 Couterier D, Roze C, Paolaggi I, Debray C. Electrical activity of the normal human stomach. Comparative study of recordings obtained from the serosal and mucosal sides. Am J Dig Dis 1972; 17: 969-76.

34 Geldof H, Van der Schee EJ, Grashuis JL. Electrogastrographic characteristics of interdigestive migrating complex in humans. Am J Physiol 1986; 250: G165-G171.

35 Pfaffenbach B, Adamek RJ, Kuhn K, Wegener M. Electrogastrography in health subjects. Dig Dis Sci 1995;40: 1445-50.

36 Parkman HP, Harris AD, Miller MA, Fisher RS. Influence of age, gender, and menstrual cycle on the normal electrogastrogram. Am J Gastroenterol 1996; 91: 127-33.

37 Lin Z, Eaker EY, Sarosiek I, McCallum RW. Gastric myoelectrical activity and gastric emptying in patients 
with functional dyspepsia. Am J Gastroenterology 1999; 94: 2384-89.

38 Koch KL, Bingaman S, Tan L, Stern RM. Visceral perceptions and gastric myoelectrical activity in healthy women and in patients with bulimia nervosa. Neurogastroenterol Motil 1998; 10: 3-10.

39 Chen JCZ, Lin Z, Pan J, McCallum RW. Abnormal gastric myoelectrical activity and delayed gastric emptying in patients with symptoms suggestive of gastroparesis. Dig Dis Sci 1996; 41: 1538-45.

40 Koch KL, Medina M, Xu L, Bingaman S, Stern RM. Gastric myoelectric responses to physiological stomach distention in humans. Gastroenterology 1994; 106: A525. (abstract)

41 Mintchev MP, Kingma YJ, Bowes KL. Accuracy of cutaneous recordings of gastric electrical activity. Gastroenterology 1993; 104: 1273-80.

42 Malagelada JR, Stanghellini V. Manometric evaluation of functional upper gut symptoms. Gastroenterology 1985; 88: $1223-31$.

43 Parkman HP, Miller MA, Trate D et al. Electrogastrography and gastric emptying scintigraphy are complementary for assessment of dyspepsia. J Clin Gastroenterol 1997; 24: 214-9.

44 Geldoff $\mathrm{H}$, der Schee EJ, van Blankenstein M, Grashuis HL. Electrogastrographic study of gastric myoelectrical activity in patients with unexplained nausea and vomiting. Gut 1986; 27: 799-808.

45 Koch KL. Dyspepsia of unknown origin - pathophysiology, diagnosis, and treatment. Dig Dis 1997; 15: 316-29.

46 Ravelli AM, Milla PJ. Vomiting and gastroesophageal motor activity in children with disorders of the central nervous system. I Ped Gastroenterol Nutr 1998; 26: 56-63.

47 Redmond JM, Smith GW, Barofsky I, Ratych RE, Goldsborough DC, Schuster MM. Physiological tests to predict long-term outcome of total abdominal colectomy for intractable constipation. Am I Gastroenterol 1995; 90: 748-53.

48 Brzana RJ, Koch KL, Bingaman S. Gastric myoelectrical activity in patients with gastric outlet obstruction and idiopathic gastroparesis. Am J Gastroenterol 1998; 93: 1803-9.

49 Rothstein RD, Alavi A, Reynolds JC. Electrogastrography in patients with gastroparesis and effect of long-term cisapride. Dig Dis Sci 1993; 38: 1518-24.

50 Cucchiara S, Minella R, Riezzo G et al. Reversal of gastric electrical dysrhythmias by cisapride in children with functional dyspepsia. Dig Dis Sci 1992; 37: 1136-40.

51 Chen JDZ, Ke MY, Lin XM, Wang Z, Zhang M. Cisapride provides symptomatic relief in functional dyspepsia associated with gastric myoelectrical abnormality. Aliment Pharmacol Ther 2000; 14: 1041-7.

52 Besherdas K, Leahy A, Mason I, Harbord M, Epstein O. The effect of cisapride on dyspepsia symptoms and the electrogastrogram in patients with non-ulcer dyspepsia. Aliment Pharmacol Ther 1998; 12: 755-9.

53 Davis $\mathrm{RH}$, Clench $\mathrm{MH}$, Mathias JR. Effects of domperidone in patients with chronic unexplained upper gastrointestinal symptoms: a double-blind, placebo-controlled study. Dig Dis Sci 1988; 33: 1505-11.

54 Bortolotti M. Electrogastrography: a seductive promise, only partially kept. Am I Gastroenterology 1998; 93: 1791-4.

55 Hasler WL, Kim MS, Chey WD, Stevenson V, Stein B, Owyang C. Central cholinergic and $\alpha$-adrenergic mediation of gastric slow wave dysrhythmias evoked during motion sickness. Am J Physiol 1995; 268: G539-G547.

56 Kim MS, Chey WD, Owyang C, Hasler WL. Role of vasopressin as a mediator of nausea and gastric slow wave dysrhythmias in motion sickness. Am J Physiol 1997; 272: G853-G862.

57 Walsh JW, Hasler WL, Nugent CE, Owyang C. Progesterone and estrogen are potential mediators of gastric slow wave dysrhythmias in the nausea of pregnancy. Am I Physiol 1996; 270: G506-G514.

58 Pimentel M, Sam C, Lin HC. Indomethacin improves symptoms and electrogastrographic findings in patients with gastric dysrhythmias. Neurogastroenterol Motil 2001; 13: 422. (abstract)

59 Bonapace ES, Parkman HP, Fisher RS. Edrophonium provocative testing during electrogastrography (EGG)-effects on dyspeptic symptoms and the EGG. Dig Dis Sci 1998; 43: $1494-500$.

60 Mintchev MP, Otto SJ, Bowes KL. Electrogastrography can recognize gastric electrical uncoupling in dogs. Gastroenterology 1997; 112: 2006-11.

61 Chen JDZ, Zhou X, Lin XM, Ouyang S, Liang J. Detection of gastric slow wave propagation from the cutaneous electrogastrogram. Am J Physiol 1999; 40: G424-G430.

62 Lin X, Chen JDZ. Abnormal gastric slow waves in patients with functional dyspepsia assessed by multichannel electrogastrography. Am J Physiol 2001; 280: G1370-G1375.

63 Lindberg G, Iwarzon M, Hammarlund B. 24-hour ambulatory electrogastrography in healthy volunteers. Scand J Gastroenterol 1996; 31: 658-64.

64 Hocke M, Seidel T, Sprott H, Oelzner P, Eitner K, Bosseckert $\mathrm{H}$. Ambulatory electrogastrography in patients with scleroderma, delayed gastric emptying, dyspepsia, and irritable bowel syndrome. Eur I Intern Med 2001; 12: 366-71. 